\title{
EPIGENETIC MECHANISM LINKED TO PIGMENTED SPOTS EXPLORED BY IN SILICO, IN VITRO AND IN VIVO METHODS
}

\author{
Corinne Reymermier ${ }^{1}$, Vincent Bardey ${ }^{1}$, Louis Danoux ${ }^{1}$, Nicolas Pelletier ${ }^{1}$, Catherine Bonnaud-Rosaye ${ }^{1}$, \\ Sabine Pain ${ }^{1}$, Gaëlle Saint-Auret ${ }^{2}$ and Valérie Andre-Frei ${ }^{1}$, \\ ${ }^{1}$ BASF Beauty Care Solutions France, 32 rue Saint Jean de Dieu, Lyon, France \\ 2 GENEL, 17 rue des Martyrs, Grenoble, France
}

\section{INTRODUCTION}

Pigmentation is a natural tanning process resulting in the production of melanin, within melanosomes by melanocytes, and their distribution throughout the epidermis. Tyrosinase is a key enzyme of the melanin synthesis. With aging, some pigmentary disorders appeared because of an over-production of tyrosinase in photo-exposed skin areas, triggering the appearance of pigmented spots. However, some new environmental assaults such as traffic-related air pollution were revealed as new contributors of pigmented spot formation, which opened new perspectives. Because melanin synthesis could be controlled through epigenetic mechanisms, we investigated if specific miRNA modulation could be responsible for melanin regulation.

\section{RESULTS}

We demonstrated that miR-490-3p could be a pigmentation regulator for skin pigmented spot improvement

Concept proof: Modulation of the protein synthesis

To prove that a protein synthesis is controlled by a miRNA in a cell (epigenetic regulation), we introduce a "miR-mimic" to artificially decrease the production of the protein.

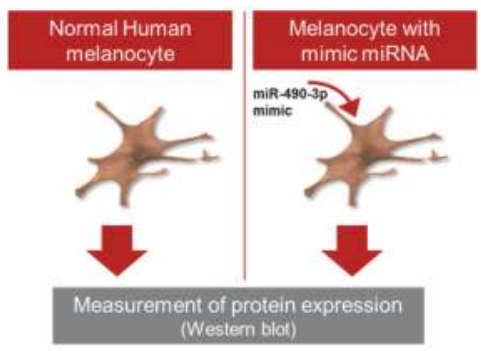

In silico analysis: miR-490-3p redundancy evaluation with Targetscan and Diana microT using specific algorithms to discover putative targets
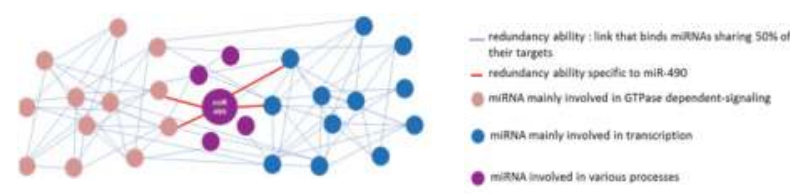

miR-490-3p position in the network in which functional relationships between miRNAs are created if they share at least 50 percent of their predicted targets.

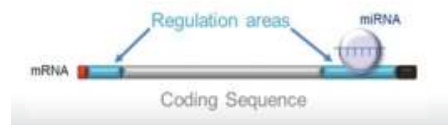

Low redundant miR-490-3p is highly specific of the tyrosinase protein synthesis

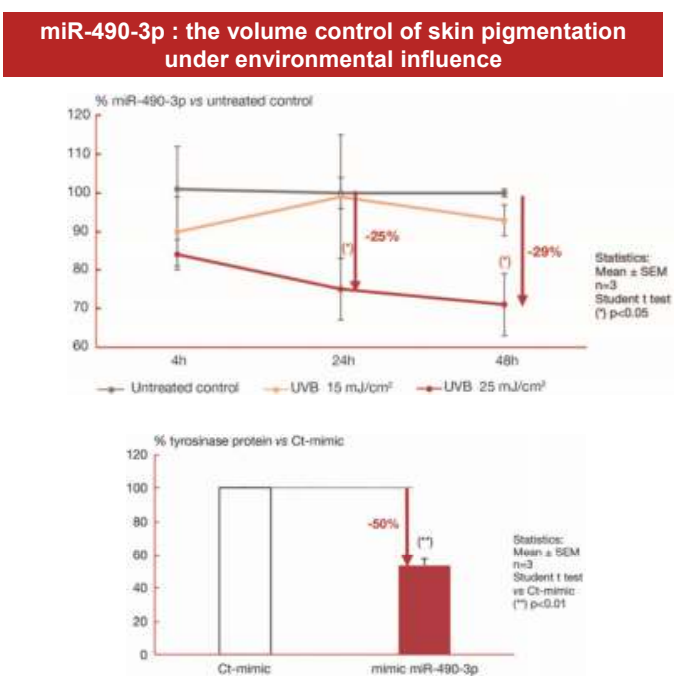

We evidenced in vitro that miR-490-3p controls TYR proteins involved in melanin synthesis
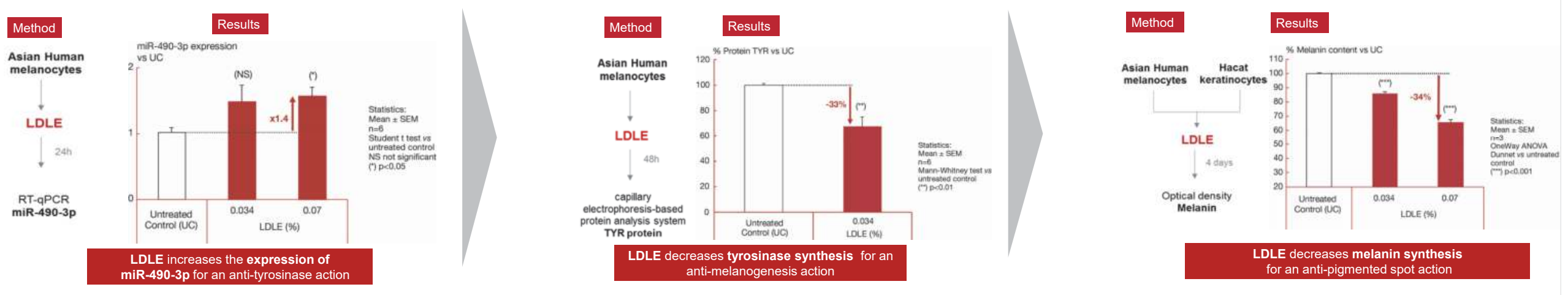

IN VIVO

Area of pigmented spots

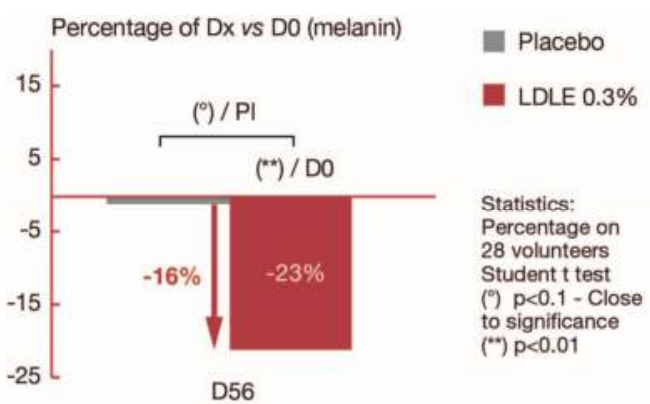

Total quantity of melanin

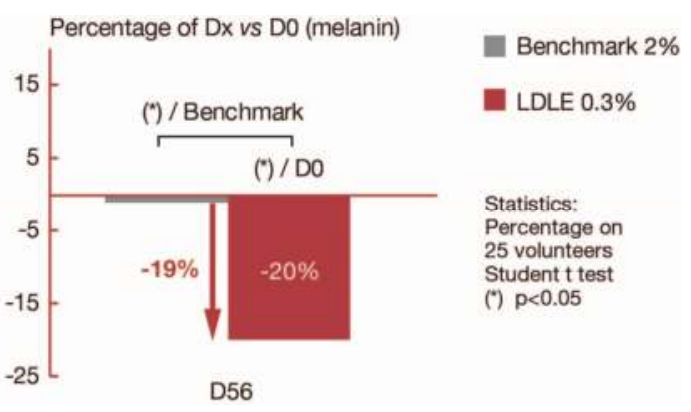

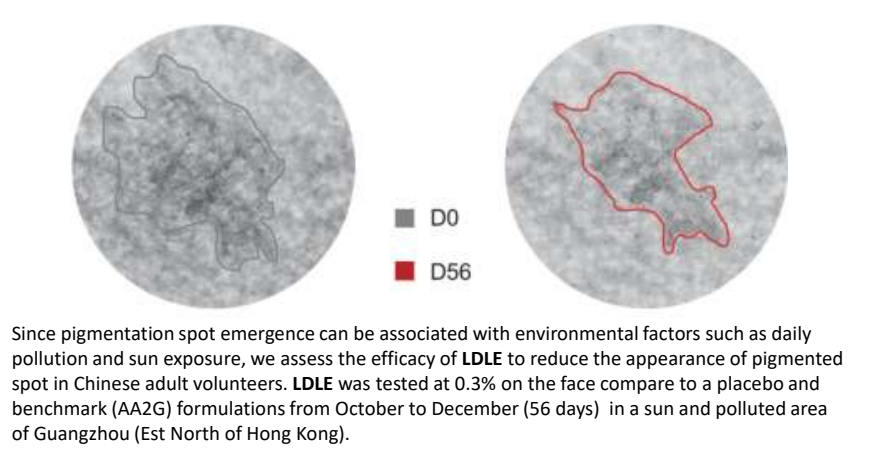

In vivo, LDLE improves pigmented spot area and melanin content at $0.3 \%$ in Chinese adult people living in a daily air-traffic pollution and sun exposed area in China.

\section{DISCUSSION}

- Based on computational algorithms from Targetscan and Diana microT, we used a new network-based method to analyze miRNA function to create functional relationship between miRNAs4. miR-490-3p showed few connections with other

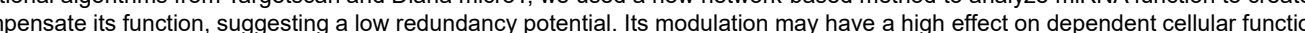

- By targeting the upregulation of this TYR regulator miR-490-3p, we aimed to reduce the skin over-pigmentation. We selected a $100 \%$ natural extract of lansium domesticum leaves, to increase the TYR regulator miR-490-3p production in

The miR-490-3p upregulation by this ingredient at $0.07 \%$ consequently decreased in 2 days the tyrosinase synthesis and in 4 days the melanin production respectively in Asian melanocytes and co-culture with keratinocytes.

Finally we demonstrated the ability of this ingredient to improve the appearance of pigmented spots versus placebo over 56 days in a clinical study on Chinese adult volunteers living in a daily air-traffic pollution and sun exposed area in China. This ingredient show better effect than AA2G (benchmark).

\section{CONCLUSION}

By stimulating the pigmented spot-related miR-490$3 p$ which is under environmental influence, Lansium domesticum leaf extract controls the skin's epigenetic pattern for reducing the pigmented spot due to daily air-traffic pollutant and sun exposed area.

\section{REFERENCES}

1. Miller M, Perspect. Public Health.136(6): 345-352,

. Krutmann J, J Dermatol. Sci. 85(3):152-161, 2017 3. Alegria-Torres JA, Epigenomics. 3(3): 267-277.

4. Bhajun R, Sci Rep. 5(12):8336, 2015

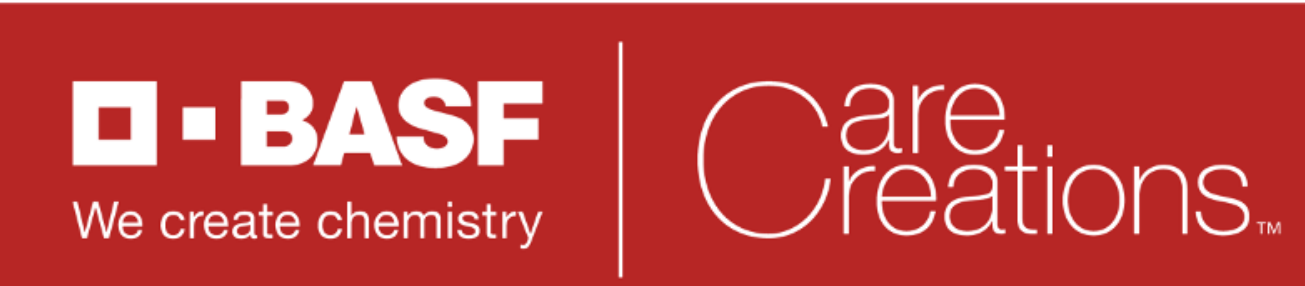

